\title{
The HELP Inequality for Hamiltonian Systems
}

\author{
B.M. BROWNa ,W.D. EVANS ${ }^{b}$ and M. MARLETTA ${ }^{c, *}$ \\ a Department of Computer Science, University of Wales, Cardiff,

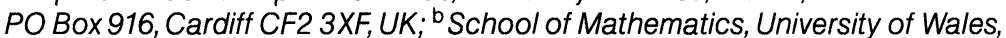 \\ Cardiff, Senghennydd Road, Cardiff, CF2 4YH, UK; ' ${ }^{\mathrm{C}}$ Department of Mathematics \\ and Computer Science, University of Leicester, University Road, \\ Leicester LE1 7RHUK
}

(Received 30 July 1998; Revised 15 September 1998)

We extend the Hardy-Everitt-Littlewood-Polya inequality, hitherto established for $2 n$th order formally selfadjoint ordinary differential equations, to a wide class of linear Hamiltonian systems. The method follows Dias (Ph.D. thesis, Cardiff: University of Wales, 1994) but without the Hilbert space setting which he uses.

Keywords: Hardy; Everitt; Littlewood; Polya; Hamiltonian system; Inequality AMS(MOS) Subject Classifications: 26D10 (26D1, 34B, 34L, 47F05)

\section{INTRODUCTION}

In the seminal paper[10]Hardy and Littlewood introduced the inequality

$$
\left(\int_{0}^{\infty}\left|f^{\prime}\right|^{2} \mathrm{~d} x\right)^{2} \leq 4 \int_{0}^{\infty}|f|^{2} \mathrm{~d} x \int_{0}^{\infty}\left|f^{\prime \prime}\right|^{2} \mathrm{~d} x
$$

which is required to hold for all functions $f$ such that the right-hand side of (1) is finite. Equality is attained when, for any $\rho>0$ and for $0 \leq x<\infty$,

$$
f(x)=A \exp (-\rho x / 2) \sin (\rho x / 2-\pi / 3) .
$$

*Corresponding author. 
No less than three proofs of (1) may be found in the book of Hardy et al. [11].

Everitt in [9] introduced the inequality

$$
\begin{aligned}
& \left(\int_{a}^{b}\left\{p(x)\left|f^{\prime}(x)\right|^{2}+(q(x)-\tau w(x))|f(x)|^{2}\right\} \mathrm{d} x\right)^{2} \\
& \quad \leq K(\tau) \int_{a}^{b} w(x)|f(x)|^{2} \mathrm{~d} x \int_{a}^{b} w(x)|\mathcal{M}[f](x)-\tau f(x)|^{2} \mathrm{~d} x
\end{aligned}
$$

where $\mathcal{M}$ is the second-order Sturm-Liouville operator

$$
\mathcal{M}[f]:=\frac{1}{w}\left(-\left(p f^{\prime}\right)^{\prime}+q f\right)
$$

with $b>a>-\infty, w(x)>0, p(x)>0, q(x) \in \mathbb{R}$ with $1 / p, q$ locally integrable and $\tau$ a real parameter. In [9] it is assumed that $\mathcal{M}$ is regular at $a$ and singular at $b$ and satisfies the so-called strong-limit-point condition. Under these conditions Everitt showed that the existence or otherwise of $K(\tau)$ in (2) depends on the spectral properties of $\mathcal{M}[f]$ and the existence criteria for (2) can be determined in terms of the behaviour of the Titchmarsh-Weyl $m$-function associated with $\mathcal{M}$. Everitt's proof in [9] is modelled on the calculus of variations proof given in [11] for (1). However an operator theoretic proof is given by Evans and Zettl in [8] and both proofs are reproduced in the article [6] of Evans and Everitt in which a neat characterisation of the criteria for a valid inequality is given in terms of the Titchmarsh-Weyl $m$-function: it is this latter approach that will serve as a model for the work reported on in this paper. The inequality (2) has been extended [7] to the case when $b$ is a regular point or $\mathcal{M}$ is in the limit-circle case at $b$. The theory associated with the existence of an analogous inequality, in which $\mathcal{M}$ is now the formally symmetric $2 N$ th order expression

$$
\mathcal{M}[f]:=\frac{1}{w} \sum_{j=0}^{N}\left(p_{j}(x) f^{(j)}\right)^{(j)},
$$

is proved by Dias in [5] (see also [2]). In this latter inequality the criteria for the existence of a valid inequality can again be determined by the spectral properties of the self-adjoint operator generated by $\mathcal{M}$ in 
$L_{w}^{2}(a, b)$ but this time it is formulated in terms of the associated Titchmarsh-Weyl $M$ matrix.

In examples of the inequality, the problem of proving the existence and also of determining the best constant is often a hard analytic problem, depending as it does on knowledge of the closed form expression for the $m$ function or $M$ matrix. A complete catalogue of all known examples to the present time is to be found in [3]. In view of the analytic difficulties in obtaining information about the best constant in particular examples, a numerical approach to the problem has been undertaken by Brown et al. This is reported in, for example, [4].

This paper reports on the development of a HELP-type inequality associated with the linear Hamiltonian system

$$
J Y^{\prime}=(\lambda \mathcal{A}+\mathcal{B}) Y
$$

where $Y$ is a $2 n$ vector and $\mathcal{A}$ and $\mathcal{B}$ are $2 n \times 2 n$ real matrices with $\mathcal{A}^{*}=\mathcal{A} \geq 0$ and $\mathcal{B}^{*}=\mathcal{B}$. $J$ is the $2 n \times 2 n$ matrix $\left(\begin{array}{cc}0 & -I \\ I & 0\end{array}\right)$ and $I$ the $n \times n$ identity matrix. It is shown in [16] that the $2 n$th order formally symmetric differential equation

$$
\mathcal{M}[f]:=\frac{1}{w} \sum_{j=0}^{n}\left(p_{j}(x) f^{(j)}\right)^{(j)}=\lambda y
$$

may be written as a Hamiltonian system, and in [1] it is shown that the matrix-vector Sturm-Liouville problem

$$
\left(P(x) Y^{\prime}\right)^{\prime}+Q(x) Y=\lambda Y
$$

where $P$ and $Q$ are $n \times n$ matrices and $Y$ is an $n$ vector may also be embedded in a Hamiltonian system. This current work is not just a simple extension of the work of Dias [5], since as $\mathcal{A}$ is semi-definite and not definite, we are unable to use the Hilbert space setting for the problem that he was able to exploit and are forced to work without this abstract structure. However the general approach of the second proof in [6] can be made to work to yield a HELP-type theory for linear Hamiltonian systems. Also examples can be found that are not covered by any previous work. 


\section{THE HAMILTONIAN SYSTEM AND THE DIRICHLET INTEGRAL}

Let $C(x)$ and $B(x)$ be real symmetric $n \times n$ matrices, and $A(x)$ an $n \times n$ real matrix, such that the elements of $A, B$ and $C$ are locally integrable over an interval $[a, \infty)$. Let $K$ be an $n \times n$ diagonal matrix of the form

$$
K=\operatorname{diag}\left(k_{1}(x), \ldots, k_{m}(x), 0,0, \ldots, 0\right),
$$

where $m \leq n$ and where the diagonal elements $k_{j}$ have locally integrable reciprocals, together with the properties

$$
\text { ess } \inf \left(k_{j}\right)>0, j=1, \ldots, m .
$$

We denote by $\tilde{K}$ the $2 n \times 2 n$ diagonal matrix whose first $m$ diagonal elements are the $k_{j}$ and whose remaining $2 n-m$ diagonal elements are all zero; we denote by $K^{\dagger}$ and $\tilde{K}^{\dagger}$ the pseudo-inverses of $K$ and $\tilde{K}$ respectively. Thus, for example,

$$
K^{\dagger}=\operatorname{diag}\left(1 / k_{1}(x), \ldots, 1 / k_{m}(x), 0,0, \ldots, 0\right) .
$$

For absolutely continuous $n$-vector functions $u$ and $v$ we define the operators $L_{1}$ and $L_{2}$ by

$$
L_{1}[u, v]:=-v^{\prime}+C(x) u-A^{\mathrm{T}}(x) v, \quad L_{2}[u, v]:=u^{\prime}-A(x) u-B(x) v .
$$

For $2 n$-vector functions $y$, partitioned in an obvious notation as

$$
y=\left(\begin{array}{c}
u_{y} \\
v_{y}
\end{array}\right)
$$

we define

$$
L(y):=\left(\begin{array}{l}
L_{1}\left[u_{y}, v_{y}\right] \\
L_{2}\left[u_{y}, v_{y}\right]
\end{array}\right)
$$

We define a bilinear form $\langle\cdot, \cdot\rangle$ by

$$
\langle f, g\rangle:=\int_{a}^{\infty} f^{*} \tilde{K} g \mathrm{~d} x=\int_{a}^{\infty} u_{f}^{*} K u_{g} \mathrm{~d} x
$$


for any $2 n$-vector functions $f$ and $g$ for which the integral converges. We also define the set of admissible functions $\mathcal{A}$ to be the set of absolutely continuous $2 n$-vector functions $f$ such that

$$
\begin{gathered}
|\langle f, f\rangle|<+\infty, \\
L_{2}\left[u_{f}, v_{f}\right]=0 \in \mathbb{R}^{n}, \\
\left(I-K K^{\dagger}\right) L_{1}\left[u_{f}, v_{f}\right]=0 \in \mathbb{R}^{n} .
\end{gathered}
$$

We make the following crucial assumption.

Assumption 1 The bilinear form $\langle\cdot, \cdot\rangle$ is positive definite on the set $\mathcal{A}$ of admissible functions $y$ which are solutions of $L(y)=\lambda \tilde{K} y$ for any $\lambda \in \mathbb{C}$.

Following Reid [15] we define the Dirichlet form associated with two $n$ vector functions $f$ and $g$ :

$$
D(f, g):=\int_{a}^{\infty}\left[v_{f}^{*} B v_{g}+u_{f}^{*} C u_{g}\right] \mathrm{d} x .
$$

Now suppose that $f, g \in \mathcal{A}$. We have

$$
\begin{aligned}
\left\langle f, \tilde{K}^{\dagger} L(g)\right\rangle= & \int_{a}^{\infty} u_{f}^{*} K\left(K^{\dagger} L_{1}\left[u_{g}, v_{g}\right]\right) \mathrm{d} x \\
= & \int_{a}^{\infty} u_{f}^{*} L_{1}\left[u_{g}, v_{g}\right] \mathrm{d} x \text { (using (12) for } g \text { ) } \\
= & \int_{a}^{\infty} u_{f}^{*}\left(-v_{g}^{\prime}+C u_{g}-A^{\mathrm{T}} v_{g}\right) \mathrm{d} x \\
= & {\left[-u_{f}^{*} v_{g}\right]_{a}^{\infty}+\int_{a}^{\infty}\left[\left(u_{f}^{\prime}\right)^{*} v_{f}+u_{f}^{*} C u_{g}-u_{f}^{*} A^{\mathrm{T}} v_{g}\right] \mathrm{d} x } \\
= & {\left[-u_{f}^{*} v_{g}\right]_{a}^{\infty}+\int_{a}^{\infty}\left[\left(A u_{f}+B v_{f}\right)^{*} v_{f}+u_{f}^{*} C u_{g}-u_{f}^{*} A^{\mathrm{T}} v_{g}\right] \mathrm{d} x } \\
& \quad\left(\text { as } L_{2}\left[u_{f}, v_{f}\right]=0\right) \\
= & {\left[-u_{f}^{*} v_{g}\right]_{a}^{\infty}+D(f, g) . }
\end{aligned}
$$

Thus we have proved the integration-by-parts formula

$$
\left\langle f, \tilde{K}^{\dagger} L(g)\right\rangle=\left[-u_{f}^{*} v_{g}\right]_{a}^{\infty}+D(f, g)
$$

for $f, g \in \mathcal{A}$. 


\section{THE HINTON-SHAW-TITCHMARSH-WEYL $M$-MATRIX}

In this section we review very briefly some results from the paper of Hinton and Shaw [12]. These will be very important in the rest of the paper.

The paper of Hinton and Shaw deals with solutions of Hamiltonian systems of differential equations of the form

$$
L(y)=\lambda \tilde{K}(x) y, \quad \lambda \in \mathbb{C} .
$$

In general, not all solutions $y$ of this equation will satisfy $\langle y, y\rangle\langle+\infty$. Those which do will lie in the set $\mathcal{A}$ of admissible functions because the structure of $\tilde{K}$ ensures that (11) and (12) are satisfied by $u_{y}, v_{y}$ when $y$ solves (14).

In addition to Assumption 1, Hinton and Shaw require the following. Assumption 2 ('Limit-point assumption') Suppose $f, g \in \mathcal{A}$. Then

$$
\lim _{x \rightarrow \infty}\left\{u_{f}(x)^{*} v_{g}(x)-v_{f}^{*}(x) u_{g}(x)\right\}=0 .
$$

Here $*$ denotes Hermitian conjugation.

Theorem 3.1 (Hinton and Shaw) Suppose that $\Im \lambda \neq 0$, and that Assumptions 1 and 2 both hold. Then the differential equation (14) possesses $n$ linearly independent solutions $\psi_{1}(x, \lambda), \ldots, \psi_{n}(x, \lambda)$ such that

$$
\left\langle\psi_{j}, \psi_{j}\right\rangle<+\infty, \quad j=1, \ldots, n .
$$

Moreover, these solutions may be normalised so that if $\Psi(x, \lambda)$ is the $2 n \times n$ matrix whose columns are $\psi_{1}(x, \lambda), \ldots, \psi_{n}(x, \lambda)$ then

$$
\Psi(a, \lambda)=\left(\begin{array}{c}
-M(\lambda) \\
I
\end{array}\right),
$$

where $M(\lambda)$ is an analytic $n \times n$ matrix function of $\lambda$ (called the Titchmarsh-Weyl matrix). This matrix has the property that $\Im M(\lambda)$ is a Nevanlinna function, in the sense that

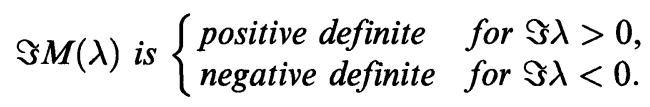


Moreover,

$$
M(\bar{\lambda})=\overline{M(\lambda)}
$$

\section{CRITERIA FOR A VALID HELP INEQUALITY}

In this section we reproduce the results of Dias [5] on existence of a HELP inequality, but in the more general context of a Hamiltonian system

$$
L(y)=\lambda \tilde{K}(x) y,
$$

or equivalently

$$
L_{1}[u, v]=\lambda K(x) u, \quad L_{2}[u, v]=0 .
$$

We assume that the following strong limit point property holds.

Assumption 3 For all functions $f, g \in \mathcal{A}$,

$$
\lim _{x \rightarrow \infty} u_{f}(x)^{*} v_{g}(x)=0 \in \mathbb{R}^{n} .
$$

Note that Assumption 3 implies Assumption 2. We define the space $\Delta_{0}$ to consist of those functions $f \in \mathcal{A}$ such that

$$
u_{f}(a)=0 \in \mathbb{R}^{n}, \quad v_{f}(a)=0 \in \mathbb{R}^{n}
$$

For $\lambda=\mu+\mathrm{i} \nu, \nu \neq 0$, we define the deficiency spaces $N_{+}(\lambda)$ and $N_{-}(\lambda)$ by

$$
N_{+}(\lambda)=\{f \in \mathcal{A} \mid L(f)=\lambda \tilde{K} f\}, \quad N_{-}(\lambda)=\{f \in \mathcal{A} \mid L(f)=\bar{\lambda} \tilde{K} f\} .
$$

As the matrices $A, B$ and $C$ occurring in the definitions of $L_{1}$ and $L_{2}$ are all real-valued, and as $\tilde{K}$ is real-valued, it is easy to see that

$$
y \in N_{+}(\lambda) \Longleftrightarrow \bar{y} \in N_{-}(\lambda) .
$$

In particular, therefore, $\operatorname{dim} N_{+}(\lambda)=\operatorname{dim} N_{-}(\lambda)$. Recall that the matrix $\Psi(x ; \lambda)$ introduced in Section 3 has columns which form a basis of $N_{+}(\lambda)$. 
Thus any function $h$ in $N_{+}(\lambda)$ may be expressed as a linear combination of the columns of $\Psi(\cdot ; \lambda)$,

$$
h=\Psi(\cdot ; \lambda) \mathbf{a},
$$

where $\mathbf{a}$ is an $n$-vector of constants depending only on $\lambda$.

We shall establish HELP inequalities for functions $f$ in the set

$$
\Delta:=\Delta_{0} \oplus N_{+}(\lambda) \oplus N_{-}(\lambda) .
$$

The first result we require is that, despite the appearance of $\lambda$ on the right hand side of (23), $\Delta$ does not depend on $\lambda$.

LeMMA $4.1 \quad \Delta=\mathcal{A}$.

Proof It is clear that $\Delta \subseteq \mathcal{A}$ so we just need to prove the opposite inclusion.

Following the notation of Section 3, we form the $2 n \times n$ matrix $\Psi$ whose columns are solutions of $L(y)=\lambda \tilde{K} y$, subject to the initial conditions

$$
\Psi(a ; \lambda)=\left(\begin{array}{c}
-M(\lambda) \\
I
\end{array}\right) .
$$

It is not difficult to see that the columns of $\Psi(\cdot ; \lambda)$ span $N_{+}(\lambda)$ and the columns of $\Psi(\cdot ; \bar{\lambda})$ span $N_{-}(\lambda)$. From Theorem 3.1 we also know that $M(\bar{\lambda})=\overline{M(\lambda)}$ and hence

$$
\Psi(x ; \bar{\lambda})=\overline{\Psi(x ; \lambda)}
$$

Thus

$$
(\Psi(a ; \lambda), \Psi(a ; \bar{\lambda}))\left(\begin{array}{cc}
\left(\frac{1}{2 i}\right) I & \frac{1}{2} I \\
-\left(\frac{1}{2 i}\right) I & \frac{1}{2} I
\end{array}\right)=\left(\begin{array}{cc}
-\Im M(\lambda) & -\Re M(\lambda) \\
0 & I
\end{array}\right) .
$$

Hence the $2 n \times 2 n$ matrix $(\Psi(a ; \lambda), \Psi(a ; \bar{\lambda}))$ is of full rank if and only if $\Im M(\lambda)$ is nonsingular. From Hinton and Shaw [12] we actually know that $\Im M(\lambda)$ is positive definite for $\Im \lambda>0$ and negative definite for $\Im \lambda<0$, which certainly therefore implies that $(\Psi(a ; \lambda), \Psi(a ; \bar{\lambda}))$ is of full rank. Thus given any function $f \in \mathcal{A}$ we can choose a $2 n$-vector

$$
\mathbf{c}=\left(\begin{array}{l}
\mathbf{c}_{1} \\
\mathbf{c}_{2}
\end{array}\right)
$$


such that

$$
f(a)=\left(\begin{array}{c}
u_{f}(a) \\
v_{f}(a)
\end{array}\right)=(\Psi(a ; \lambda), \Psi(a ; \bar{\lambda})) \mathbf{c}=\Psi(a ; \lambda) \mathbf{c}_{1}+\Psi(a ; \bar{\lambda}) \mathbf{c}_{2}
$$

Defining

$$
g(x)=f(x)-\Psi(x ; \lambda) \mathbf{c}_{1}-\Psi(x ; \bar{\lambda}) \mathbf{c}_{2},
$$

it is clear that $g$ is an element of $\mathcal{A}$ such that

$$
u_{g}(a)=v_{g}(a)=0 \in \mathbb{R}^{n}
$$

Thus $g \in \Delta_{0}$. Also, $\Psi(\cdot ; \lambda) \mathbf{c}_{1} \in N_{+}(\lambda)$ and $\Psi(x ; \bar{\lambda}) \mathbf{c}_{2} \in N_{-}(\lambda)$. Hence $f \in \Delta$, which completes the proof.

Remark This result is essentially the Von Neumann decomposition formula - see [13, Lemma 10.2.5]. We have been forced to prove it in this very direct way by the fact that there does not appear to be a natural Hilbert space setting for these problems, except in special cases.

Lemma 4.1 establishes the decomposition

$$
\mathcal{A}=\Delta_{0} \oplus N_{+}(\lambda) \oplus N_{-}(\lambda) .
$$

We shall use this extensively in the sequel.

LEMMA 4.2 The direct sum appearing in (25) is an orthogonal sum with respect to the sesquilinear form

$$
\langle f, g\rangle_{\lambda}:=\left\langle\tilde{K}^{\dagger}(L(f)-\mu \tilde{K} f), \tilde{K}^{\dagger}(L(g)-\mu \tilde{K} g)\right\rangle+\nu^{2}\langle f, g\rangle .
$$

Proof Suppose that $f \in \Delta_{0}$ and $g \in N_{+}(\lambda)$, so that $L(g)=\mu \tilde{K} g+\mathrm{i} \nu \tilde{K} g$. Then

$$
\begin{aligned}
\langle f, g\rangle_{\lambda}= & \left\langle\tilde{K}^{\dagger}(L(f)-\mu \tilde{K} f), \mathrm{i} \nu \tilde{K} g\right\rangle+\nu^{2}\langle f, g\rangle \\
= & \mathrm{i} \nu \int_{a}^{\infty}\left(L_{1}\left[u_{f}, v_{f}\right]\right)^{*} K^{\dagger} K K^{\dagger} K u_{g}-\mathrm{i} \mu \nu \int_{a}^{\infty} u_{f}^{*} K K^{\dagger} K K^{\dagger} K u_{g} \\
& +\nu^{2} \int_{a}^{\infty} u_{f}^{*} K u_{g} .
\end{aligned}
$$


We now use (12) to simplify the first integral, and the result $K K^{\dagger} K=K$ to simplify the second. These yield

$$
\langle f, g\rangle_{\lambda}=\mathrm{i} \nu \int_{a}^{\infty} L_{1}\left[u_{f}, v_{f}\right]^{*} u_{g}-\mathrm{i} \lambda \nu \int_{a}^{b} u_{f}^{*} K u_{g}
$$

Using the integration-by-parts formula (13) twice, together with (20) and (21) to eliminate the boundary terms, we obtain

$$
\int_{a}^{\infty} L_{1}\left[u_{f}, v_{f}\right]^{*} u_{g}=\int_{a}^{\infty} u_{f}^{*} L_{1}\left[u_{g}, v_{g}\right]
$$

Now $L(g)=\lambda \tilde{K} g$ implies that $L_{1}\left[u_{g}, v_{g}\right]=\lambda K u_{g}$; substituting this back into (28) gives

$$
\int_{a}^{\infty} L_{1}\left[u_{f}, v_{f}\right]^{*} u_{g}=\lambda \int_{a}^{\infty} u_{f}^{*} K u_{g}
$$

Substituting (29) into (27) yields

$$
\langle f, g\rangle_{\lambda}=0,
$$

as required.

Similarly one may prove that $\langle f, g\rangle=0$ for $f \in \Delta_{0}$ and $g \in N_{-}(\lambda)$. It remains to show that $\langle f, g\rangle=0$ for $f \in N_{+}(\lambda)$ and $g \in N_{-}(\lambda)$. This case is easy: we have $L(f)=\lambda \tilde{K} f$ and $L(g)=\lambda \tilde{K} g$, so that

$$
\begin{aligned}
\langle f, g\rangle_{\lambda} & =\left\langle\mathrm{i} \nu \tilde{K}^{\dagger} \tilde{K} f, \mathrm{i} \nu \tilde{K}^{\dagger} \tilde{K} g\right\rangle+\nu^{2}\langle f, g\rangle \\
& =-\nu^{2} \int_{a}^{\infty} u_{f}^{*} K K^{\dagger} K K K^{\dagger} u_{g}+\nu^{2} \int_{a}^{\infty} u_{f}^{*} K u_{g}=0,
\end{aligned}
$$

since $K K^{\dagger} K=K$. This completes the proof.

LEMMA 4.3

$$
\langle\Psi, \Psi\rangle_{\lambda}=2 \nu \Im(M(\lambda))
$$


Proof Using the definition of $\langle\cdot, \cdot\rangle_{\lambda}$ we have

$$
\langle\Psi, \Psi\rangle_{\lambda}=\left\langle\tilde{K}^{\dagger}(L(\Psi)-\mu \tilde{K} \Psi), \tilde{K}^{\dagger}(L(\Psi)-\mu \tilde{K} \Psi)\right\rangle+\nu^{2}\langle\Psi, \Psi\rangle .
$$

Now we use the fact that $L(\Psi)=(\mu+\mathrm{i} \nu) \tilde{K} \Psi$ to obtain

$$
\langle\Psi, \Psi\rangle_{\lambda}=\nu^{2} \int_{a}^{\infty}\left(\tilde{K}^{\dagger} \tilde{K} \Psi\right)^{*} K \tilde{K}\left(\tilde{K}^{\dagger} \tilde{K} \Psi\right)+\nu^{2}\langle\Psi, \Psi\rangle
$$

From the identity $\tilde{K} \tilde{K}^{\dagger} \tilde{K}=\tilde{K}$ this simplifies to give

$$
\langle\Psi, \Psi\rangle_{\lambda}=2 \nu^{2}\langle\Psi, \Psi\rangle
$$

But now

$$
\begin{aligned}
\langle\Psi, \Psi\rangle & =\int_{a}^{\infty} \Psi^{*} \tilde{K} \Psi=\frac{1}{\lambda} \int_{a}^{\infty} \Psi^{*} L(\Psi) \\
& =\frac{1}{\lambda}\left\{u_{\Psi}^{*}(a) v_{\Psi}(a)+D(\Psi, \Psi)\right\},
\end{aligned}
$$

the last identity following from the integration-by-parts formula (13). From the initial conditions on $\Psi$, we have $u_{\Psi}(a)=-M(\lambda)$ and $v_{\Psi}(a)=I$, yielding

$$
\langle\Psi, \Psi\rangle=\frac{1}{\lambda}\left\{-M^{*}(\lambda)+D(\Psi, \Psi)\right\}
$$

Multiplying both sides by $\lambda$ and taking the imaginary parts yields

$$
\nu\langle\Psi, \Psi\rangle=-\Im\left(M^{*}(\lambda)\right)=\Im(M(\lambda)),
$$

the last equality on the right following from the well known fact that $M$ is a symmetric matrix [12]. Combining (31) and (32) gives the required result.

LEMMA 4.4 Let $f \in \mathcal{A}$ be a real-valued function, and suppose that $f=f_{0}+h+\bar{h}$, where $f_{0} \in \Delta_{0}$ and $h \in N_{+}(\lambda), \bar{h} \in N_{-}(\lambda)$. Suppose that

$$
h=\Psi \mathbf{a},
$$


where $\mathbf{a}=\left(a_{1}, \ldots, a_{n}\right)$ is a constant vector. Then

$$
\langle f, f\rangle_{\lambda}=\left\langle f_{0}, f_{0}\right\rangle_{\lambda}+4 \nu \mathbf{a}^{*} \Im(M(\lambda)) \mathbf{a} .
$$

Proof From Lemma 4.2 we know that the $\operatorname{sum} f_{0}+h+\bar{h}$ is orthogonal with respect to $\langle\cdot, \cdot\rangle_{\lambda}$. Thus we have

$$
\langle f, f\rangle_{\lambda}=\left\langle f_{0}, f_{0}\right\rangle_{\lambda}+\langle h, h\rangle_{\lambda}+\langle\bar{h}, \bar{h}\rangle_{\lambda}=\left\langle f_{0}, f_{0}\right\rangle_{\lambda}+2\langle h, h\rangle_{\lambda} .
$$

From (33), it is clear that

$$
\langle h, h\rangle_{\lambda}=\mathbf{a}^{*}\langle\Psi, \Psi\rangle_{\lambda} \mathbf{a}
$$

Using (30) yields

$$
\langle h, h\rangle_{\lambda}=2 \nu \mathbf{a}^{*} \Im(M(\lambda)) \mathbf{a} .
$$

Combining (35) and (36) gives the required result.

For real-valued $f \in \mathcal{A}$ consider $\langle f, f\rangle_{\lambda}$. From (26) we have

$$
\begin{aligned}
\langle f, f\rangle_{\lambda}= & \left\langle\tilde{K}^{\dagger} L(f), \tilde{K}^{\dagger} L(f)\right\rangle-2 \mu\left\langle\tilde{K}^{\dagger} \tilde{K} f, \tilde{K}^{\dagger} L(f)\right\rangle \\
& +\mu^{2}\left\langle\tilde{K}^{\dagger} \tilde{K} f, \tilde{K}^{\dagger} \tilde{K} f\right\rangle+\nu^{2}\langle f, f\rangle \\
= & \left\langle\tilde{K}^{\dagger} L(f), \tilde{K}^{\dagger} L(f)\right\rangle-2 \mu\left\langle f, \tilde{K}^{\dagger} L(f)\right\rangle+|\lambda|^{2}\langle f, f\rangle,
\end{aligned}
$$

where we have used the identity $\tilde{K} \tilde{K}^{\dagger} \tilde{K}=\tilde{K}$ several times. Now we apply the integration-by-parts formula (13) to the term $\left\langle f, \tilde{K}^{\dagger} L(f)\right\rangle$ to obtain

$$
\langle f, f\rangle_{\lambda}=\left\langle\tilde{K}^{\dagger} L(f), \tilde{K}^{\dagger} L(f)\right\rangle-2 \mu D(f, f)+|\lambda|^{2}\langle f, f\rangle-2 \mu u_{f}(a)^{*} v_{f}(a)
$$

We define the form

$$
J(f):=\left\langle\tilde{K}^{\dagger} L(f), \tilde{K}^{\dagger} L(f)\right\rangle-2 \mu D(f, f)+|\lambda|^{2}\langle f, f\rangle .
$$

Then (37) immediately yields

$$
J(f)=\langle f, f\rangle_{\lambda}+2 \mu u_{f}(a)^{*} v_{f}(a)
$$


As $f=f_{0}+h+\bar{h}$ with $f_{0} \in \Delta_{0}$, Eq. (21) yields $u_{f}(a)=2 \Re\left(u_{h}(a)\right)$ and $v_{f}(a)=2 \Re\left(v_{h}(a)\right)$. Equations (24) and (33) give

$$
u_{h}(a)=-M(\lambda) \mathbf{a}, \quad v_{h}(a)=\mathbf{a} .
$$

Substituting into (39) we obtain

$$
J(f)=\langle f, f\rangle_{\lambda}-8 \mu(\Re(\mathbf{a}))^{\mathrm{T}} \Re(M(\lambda) \mathbf{a}) .
$$

Combining this with (34) yields

$$
J(f)=\left\langle f_{0}, f_{0}\right\rangle_{\lambda}+4 \nu \mathbf{a}^{*} \Im(M(\lambda)) \mathbf{a}-8 \mu(\Re(\mathbf{a}))^{\mathrm{T}} \Re(M(\lambda) \mathbf{a}) .
$$

We now separate $M(\lambda)$ and a into real and imaginary parts, denoted by

$$
M(\lambda)=M_{\mathrm{R}}+\mathrm{i} M_{\mathrm{I}}, \quad \mathbf{a}=\mathbf{a}_{\mathrm{R}}+\mathbf{i} \mathbf{a}_{\mathrm{I}} .
$$

Some simple algebra shows that (42) may be rearranged in the form

$$
J(f)=\left\langle f_{0}, f_{0}\right\rangle_{\lambda}+\frac{4}{\nu}\left(\nu \mathbf{a}_{\mathrm{I}}+\mu \mathbf{a}_{\mathrm{R}}\right)^{\mathrm{T}} M_{\mathrm{I}}\left(\nu \mathbf{a}_{\mathrm{I}}+\mu \mathbf{a}_{\mathrm{R}}\right)+\frac{4}{\nu} \mathbf{a}_{\mathrm{R}}^{\mathrm{T}} \Im\left(-\lambda^{2} M(\lambda)\right) \mathbf{a}_{\mathrm{R}} .
$$

In order to make further progress we must introduce a further assumption about the set $\mathcal{A}$ of admissible functions.

Assumption 4 For all non-zero $f \in \mathcal{A}$,

$$
\langle f, f\rangle>0 .
$$

With this assumption we can rewrite the expression (38) as a quadratic form in $\rho:=|\lambda|$. Let $\lambda=\rho \mathrm{e}^{\mathrm{i} \phi}$ so that $\mu=\rho \cos \phi$. Then some simple algebra shows that

$$
\begin{aligned}
J(f)= & J_{\phi}(f):=\langle f, f\rangle\left(\rho-\frac{D(f, f) \cos \phi}{\langle f, f\rangle}\right)^{2} \\
& +\left\langle\tilde{K}^{\dagger} L(f), \tilde{K}^{\dagger} L(f)\right\rangle-\frac{D(f, f) \cos ^{2} \phi}{\langle f, f\rangle} .
\end{aligned}
$$


Similarly, whenever $\lambda=\rho \mathrm{e}^{\mathrm{i}(\phi+\pi)}$, we obtain

$$
\begin{aligned}
J(f)= & J_{\phi+\pi}(f):=\langle f, f\rangle\left(\rho+\frac{D(f, f) \cos \phi}{\langle f, f\rangle}\right)^{2} \\
& +\left\langle\tilde{K}^{\dagger} L(f), \tilde{K}^{\dagger} L(f)\right\rangle-\frac{D(f, f) \cos ^{2} \phi}{\langle f, f\rangle}
\end{aligned}
$$

Given any non-zero $f$ and any $\phi \in[0, \pi / 2)$ one may always choose

$$
\rho=\left|\frac{D(f, f) \cos \phi}{\langle f, f\rangle}\right|
$$

This eliminates the first term on the right hand side of one of $(44,45)$. The remaining term will be non-negative if and only if

$$
D(f, f) \leq \sec ^{2} \phi\langle f, f\rangle\left\langle\tilde{K}^{\dagger} L(f), \tilde{K}^{\dagger} L(f)\right\rangle .
$$

This is clearly a HELP inequality, provided $0 \leq \phi<\pi / 2$. We are now in a position to prove the following theorem.

THEOREM 4.5 Let $\mathcal{S}$ be the set of all values of $\phi \in(0, \pi / 2]$ such that the following two conditions hold:

$$
\left.\begin{array}{rl}
\Im\left(-\lambda^{2} M(\lambda)\right) \geq 0 & \left(\lambda=\rho \mathrm{e}^{\mathrm{i} \phi}\right) \\
\Im\left(\lambda^{2} M(\lambda)\right) \geq 0 & \left(\lambda=\rho \mathrm{e}^{\mathrm{i}(\phi+\pi)}\right)
\end{array}\right\} \quad \forall \rho>0 .
$$

Then $\pi / 2 \in \mathcal{S}$. Let $\theta_{0}=\inf (\mathcal{S})$. Then a HELP inequality

$$
D(f, f) \leq \kappa\langle f, f\rangle\left\langle\tilde{K}^{\dagger} L(f), \tilde{K}^{\dagger} L(f)\right\rangle
$$

holds for all $f \in \mathcal{A}$ if and only if $\theta_{0}<\pi / 2$. Moreover, the best constant $\kappa$ in the inequality is given by $\kappa=\sec ^{2} \theta_{0}$.

Proof Suppose that $\theta_{0}<\pi / 2$. Choose $\phi=\theta_{0}$. Given $f$, choose $\rho$ according to (46), eliminating the first term on the right hand side of one of $(44,45)$. The conditions (48) then imply that (47) holds, giving a HELP inequality with $\kappa=\sec ^{2} \theta_{0}$. Thus the condition $\theta_{0}<\pi / 2$ is sufficient for a HELP inequality. 
To see that $\kappa=\sec ^{2} \theta_{0}$ is the best constant, suppose we look for a smaller constant $\hat{\kappa}=\sec ^{2} \phi$ for some $\phi \in\left(0, \theta_{0}\right)$. By definition of $\theta_{0}$ there will exist some $\rho>0$ such that at least one of the following conditions fails:

$$
\begin{gathered}
\Im\left(-\lambda^{2} M(\lambda)\right) \geq 0, \quad \lambda=\rho \mathrm{e}^{\mathrm{i} \phi}, \\
\Im\left(\lambda^{2} M(\lambda)\right) \geq 0, \quad \lambda=\rho \mathrm{e}^{\mathrm{i}(\phi+\pi)} .
\end{gathered}
$$

Suppose the first condition fails. Then we can find a real vector $\mathbf{a}_{\mathbf{R}}$ such that

$$
\mathbf{a}_{\mathbf{R}}^{\mathrm{T}} \Im\left(-\lambda^{2} M(\lambda)\right) \mathbf{a}_{\mathbf{R}}<0 .
$$

With $\mu=\rho \cos \theta, \nu=\rho \sin \theta$, we choose a real vector $\mathbf{a}_{\mathbf{I}}=-\mu / \nu \mathbf{a}_{\mathbf{R}}$. Next we define $h=\Psi\left(\mathbf{a}_{\mathbf{R}}+\mathbf{i} \mathbf{a}_{\mathrm{I}}\right)$ and $f=h+\bar{h}$ so that from (43) we have $J(f)<0$. From (44) this shows that the HELP inequality fails with $\kappa=\sec ^{2} \phi$. This covers the case where (50) does not hold. A similar argument deals with the case where (51) does not hold, so the choice $\kappa=\sec ^{2} \theta$ is clearly best-possible. This also proves that the condition $\theta_{0}<\pi / 2$ is necessary for a HELP inequality to hold.

\section{GENERALISATIONS}

It would be nice to remove Assumption 4, and indeed we can do this for at least part of the result in Theorem 4.5. If Assumption 4 is removed then the condition $\theta_{0}<\pi / 2$ of Theorem 4.5 still implies a HELP inequality for those nonzero functions $f \in \mathcal{A}$ such that $\langle f, f\rangle>0$.

What happens if $f$ is a nonzero function such that $\langle f, f\rangle=0$ ? In this case (38) loses the term $|\lambda|^{2}\langle f, f\rangle$ on the right hand side. Equation (43) still holds, and so the condition $\theta_{0}<\pi / 2$ implies positive-definiteness of

$$
J(f)=\left\langle\tilde{K}^{\dagger} L(f), \tilde{K}^{\dagger} L(f)\right\rangle-2 \mu D(f, f) .
$$

We can take $\mu$ to be arbitrarily large and of either sign in (38) and so we deduce that, when $\theta_{0}<\pi / 2$,

$$
\langle f, f\rangle=0 \Longrightarrow D(f, f)=0 .
$$

Thus we still have a HELP inequality in this case, albeit a trivial one. 


\section{EXAMPLES}

We present three examples of HELP inequalities associated with Hamiltonian systems. Two of these may be derived from HELP inequalities for even-order differential operators; one, however, is completely new. First, however, we require a technical lemma.

LEMMA 6.1 (Compactness Lemma) Suppose that there exists $\phi \in(0, \pi / 2)$ such that the conditions

$$
\begin{aligned}
& \Im\left(-\lambda^{2} M(\lambda)\right)>0, \quad \lambda=\rho \mathrm{e}^{\mathrm{i} \theta}, \\
& \Im\left(\lambda^{2} M(\lambda)\right)>0, \quad \lambda=-\rho \mathrm{e}^{\mathrm{i} \theta}
\end{aligned}
$$

hold for all $\theta \in[\phi, \pi / 2]$, for all sufficiently large $\rho$ and for all sufficiently small $\rho$. Then, in the notation of Theorem $4.5, \theta_{0}<\pi / 2$, and so a HELP inequality holds.

Proof The Nevanlinna property of $M(\lambda)$ ensures that (52) holds for $\theta=\pi / 2$. Hence by continuity, for each $\rho>0$ there exists $\phi_{\rho} \in(0, \pi / 2)$ such that (52) holds for any $\theta \in\left[\phi_{\rho}, \pi / 2\right]$. We can choose $\phi_{\rho}$ as a continuous function of $\rho$. Thus given any set $\left[\rho_{\min }, \rho_{\max }\right]$, we can choose $\phi_{*} \in(0, \pi / 2)$ such that $\Im\left(-\lambda^{2} M(\lambda)\right)>0$ for $\lambda=\rho \mathrm{e}^{\mathrm{i} \theta}$, for $\theta \in\left[\phi_{*}, \pi / 2\right]$ and $\rho \in\left[\rho_{\min }, \rho_{\max }\right]$. However, by hypothesis, we can choose $\rho_{\min }$ sufficiently small and $\rho_{\max }$ sufficiently large to ensure that (52) holds for all $\theta \in$ $[\phi, \pi / 2]$, for all $\rho \in\left(0, \rho_{\min }\right] \cup\left[\rho_{\max }, \infty\right)$. Hence (52) holds for all $\theta \in\left[\min \left(\phi_{*}, \phi\right), \pi / 2\right]$ for all $\rho>0$.

A similar argument deals with (53).

\subsection{Example 1}

We consider the Hamiltonian system with $n=2$ for which, in our earlier notation,

$$
\begin{array}{ll}
C=\left(\begin{array}{ll}
0 & 0 \\
0 & 0
\end{array}\right), & A=\left(\begin{array}{ll}
0 & 1 \\
1 & 0
\end{array}\right), \\
K=\left(\begin{array}{ll}
1 & 0 \\
0 & 0
\end{array}\right), & B=\left(\begin{array}{ll}
2 & 0 \\
0 & 1
\end{array}\right) .
\end{array}
$$


In order to determine the square-integrable solutions of this system we require the eigenvalues and eigenvectors of the matrix

$$
S=\left(\begin{array}{cc}
A & B \\
-\lambda K & -A
\end{array}\right)
$$

The eigenvalues are readily seen to be $\pm \mu_{ \pm}$, where

$$
\mu_{ \pm}=\left(1-\lambda \pm \sqrt{\lambda^{2}-\lambda}\right)^{1 / 2}
$$

Here we adopt the convention that the square root has positive real part. The eigenvector associated with an eigenvalue $\mu$ is given by

$$
v=\left(\mu\left(\mu^{2}+1\right), \mu^{2}+\lambda+1,-\lambda \mu^{2}, \lambda \mu\right)^{\mathrm{T}} .
$$

Given an eigenvalue $\mu$ and eigenvector $v$ the associated solution of the Hamiltonian system is $v \exp (\mu x)$. It is easy to check that the positivedefiniteness condition in Assumption 1 holds for any linear combination of our four solutions here.

For $\lambda$ in the first quadrant of the complex plane with $|\lambda|$ large, it is easy to see that the eigenvalues with negative real part are $-\mu_{+}$and $\mu_{-}$. The associated solutions $y_{1}(x)$ and $y_{2}(x)$ of the Hamiltonian system are given by

$$
\left(y_{1}(x) y_{2}(x)\right)=\left(\begin{array}{cc}
\mu_{-}\left(\mu_{-}^{2}+1\right) \exp \left(\mu_{-} x\right) & -\mu_{+}\left(\mu_{+}^{2}+1\right) \exp \left(-\mu_{+} x\right) \\
\left(\mu_{-}^{2}+\lambda+1\right) \exp \left(\mu_{-} x\right) & \left(\mu_{+}^{2}+\lambda+1\right) \exp \left(-\mu_{+} x\right) \\
-\lambda \mu_{-}^{2} \exp \left(\mu_{-} x\right) & -\lambda \mu_{+}^{2} \exp \left(-\mu_{+} x\right) \\
\lambda \mu_{-} \exp \left(\mu_{-} x\right) & -\lambda \mu_{+}^{2} \exp \left(-\mu_{+} x\right)
\end{array}\right) .
$$

From Theorem 3.1 we know that

$$
\left(y_{1}(0) y_{2}(0)\right)=\left(\begin{array}{c}
-M(\lambda) \\
I
\end{array}\right) V
$$

for some non-singular $n \times n$ matrix $V$. This allows us to deduce that

$$
M(\lambda)=\left(\begin{array}{cc}
\left(\mu_{-}-\mu_{+}\right) / \lambda & -\left(1+\mu_{-} \mu_{+}\right) / \lambda \\
-\left(1+\mu_{-} \mu_{+}\right) / \lambda & \left(\mu_{-}-\mu_{+}\right)(\lambda+1) / \lambda
\end{array}\right)
$$


Putting $\lambda=\rho \exp (\mathrm{i} \theta), 0<\theta \leq \pi / 2, \rho>0$, it is easy to see that $\mu_{+} \sim 1 / \sqrt{2}$ and $\mu_{-} \sim \mathrm{i} \sqrt{2} \sqrt{\rho} \exp (\mathrm{i} \theta / 2)$ for large $\rho$. This allows us to deduce that

$$
\begin{gathered}
\Im\left(-\lambda^{2} m_{11}(\lambda)\right) \sim-\sqrt{2} \rho^{3 / 2} \cos (3 \theta / 2), \\
\Im\left(-\lambda^{2} m_{22}(\lambda)\right) \sim-\sqrt{2} \rho^{5 / 2} \cos (5 \theta / 2), \\
\Im\left(-\lambda^{2} m_{12}(\lambda)\right) \sim \rho^{3 / 2} \cos (3 \theta / 2) .
\end{gathered}
$$

It is therefore easy to check that for all sufficiently large $\rho$, there exists a sector surrounding $\theta=\pi / 2$ in which $\Im\left(-\lambda^{2} M(\lambda)\right)$ is positive definite.

Next we check the case where $|\lambda|$ is small. It is easy to check that when $|\lambda|$ is small, the eigenvalues of the matrix $S$ in (55) with negative real part are $-\mu_{+}$and $-\mu_{-}$. Also,

$$
\mu_{+} \sim-1-\frac{\lambda}{2}, \quad \mu_{-} \sim 1-\frac{\mathrm{i} \sqrt{\lambda}}{2} .
$$

The $M$-matrix is obtained by replacing $\mu_{-}$by $-\mu_{-}$in (58), and hence

$$
\lambda^{2} M(\lambda) \sim\left(\begin{array}{cc}
-2 \lambda & \lambda^{2} / 2 \\
\lambda^{2} / 2 & -2 \lambda
\end{array}\right)
$$

Putting $\lambda=\rho \exp (\mathrm{i} \theta)$ we get

$$
-\lambda^{2} M(\lambda)=\left(\begin{array}{cc}
2 \rho \sin \theta & -\rho^{2} / 2 \sin 2 \theta \\
-\rho^{2} / 2 \sin 2 \theta & 2 \rho \sin \theta
\end{array}\right) .
$$

Again it is easy to check that for all sufficiently small $\rho$, there exists a sector surrounding $\theta=\pi / 2$ in which this matrix is positive definite.

We must now check the case where $\lambda=\rho \exp (\mathrm{i}(\theta+\pi)), 0<\theta \leq \pi / 2$ : again we must consider separately the cases of large and small $\rho$. We have

$$
\mu_{ \pm}=\left(1+\rho \mathrm{e}^{\mathrm{i} \theta} \pm \sqrt{\rho^{2} \mathrm{e}^{2 \mathrm{i} \theta}+\rho \mathrm{e}^{\mathrm{i} \theta}}\right)^{1 / 2},
$$

and it is easy to check that, both in the case of small $\rho$ and in the case of large $\rho,-\mu_{+}$and $-\mu_{-}$are the eigenvalues of $S$ with negative real part. 
Thus, changing $\mu_{-}$to $-\mu_{-}$in (58),

$$
M(\lambda)=\left(\begin{array}{cc}
-\left(\mu_{+}+\mu_{-}\right) / \lambda & -\left(1-\mu_{+} \mu_{-} / \lambda\right) \\
-\left(1-\mu_{+} \mu_{-}\right) / \lambda & -[(\lambda+1) / \lambda]\left(\mu_{+}+\mu_{-}\right)
\end{array}\right) .
$$

For large $\rho$ we have

$$
\mu_{-} \sim 1, \quad \mu_{+} \sim \sqrt{2} \sqrt{\rho} \exp (\mathrm{i} \theta)
$$

while for small $\rho$

$$
\mu_{ \pm} \sim 1 \pm \frac{1}{2} \sqrt{\rho} \mathrm{e}^{\mathrm{i} \theta / 2}
$$

Hence it is easily shown that for large $\rho$,

$$
\Im\left(\lambda^{2} M(\lambda)\right) \sim \sqrt{2} \rho^{3 / 2}\left(\begin{array}{cc}
\sin (3 \theta / 2) & -\sin (3 \theta / 2) \\
-\sin (3 \theta / 2) & -\rho \sin (5 \theta / 2)
\end{array}\right) .
$$

It is easily checked that for all sufficiently large $\rho$ there exists a sector surrounding $\theta=\pi / 2$ in which this is positive definite.

For small $\rho$ we obtain

$$
M(\lambda) \sim 2 \rho \sin \theta\left(\begin{array}{cc}
1 & \frac{1}{2} \rho \cos \theta \\
\frac{1}{2} \rho \cos \theta & 1
\end{array}\right)
$$

and for all sufficiently small $\rho$ there exists a sector surrounding $\theta=\pi / 2$ in which this is positive definite.

By the compactness lemma (Lemma 6.1) we therefore have a HELP inequality for this problem, valid for all functions $f$ for which $\langle f, f\rangle>0$.

It is interesting to observe that for this Hamiltonian system, Assumption 4 fails. The function

$$
f(x)=\left(\begin{array}{c}
0 \\
\mathrm{e}^{-x / \sqrt{2}} \\
-(1 / 2) \mathrm{e}^{-x / \sqrt{2}} \\
-(1 / \sqrt{2}) \mathrm{e}^{-x / \sqrt{2}}
\end{array}\right)
$$

is an admissible function with $\langle f, f\rangle=0$. 


\subsection{Example 2}

For our second example we consider the Hamiltonian system associated with the second order matrix-vector Sturm-Liouville equation

$$
-Y^{\prime \prime}+\left(\begin{array}{cc}
x & 0 \\
0 & -x
\end{array}\right) Y=\lambda Y, \quad x \in(0, \infty)
$$

Here $Y$ is a 2-vector. The system can be cast in the Hamiltonian form

$$
\begin{aligned}
-V^{\prime} & =\lambda U+\left(\begin{array}{cc}
x & 0 \\
0 & -x
\end{array}\right) V, \\
U^{\prime} & =V .
\end{aligned}
$$

It is easy to check that our Dirichlet form $D(f, f)$ is the same as the usual Dirichlet integral for such a problem:

$$
D(f, f)=\int_{0}^{\infty}\left\{\left\|Y^{\prime}\right\|^{2}+Y^{*}\left(\begin{array}{cc}
x & 0 \\
0 & -x
\end{array}\right) Y\right\} \mathrm{d} x, \quad \text { where } f=\left(\begin{array}{c}
Y \\
Y^{\prime}
\end{array}\right) .
$$

It is also easy to check that Assumptions 1-4 all hold.

For fixed $\lambda$ with $\Im(\lambda) \neq 0$ let $y_{1}(x)$ denote a nontrivial $L^{2}$ solution of the scalar equation $-y^{\prime \prime}+x y=\lambda y$ and let $y_{2}$ denote a nontrivial $L^{2}$ solution of the scalar equation $-y^{\prime \prime}-x y=\lambda y$. Then

$$
Y_{1}=\left(\begin{array}{c}
y_{1} \\
0
\end{array}\right), \quad Y_{2}=\left(\begin{array}{c}
0 \\
y_{2}
\end{array}\right)
$$

are $L^{2}$ solutions of (62). We form the matrices

$$
U(x)=\left(\begin{array}{cc}
y_{1}(x) & 0 \\
0 & y_{2}(x)
\end{array}\right), \quad V(x)=\left(\begin{array}{cc}
y_{1}^{\prime}(x) & 0 \\
0 & y_{2}^{\prime}(x)
\end{array}\right)
$$

and observe that $\left(\begin{array}{l}U \\ V\end{array}\right)$ specifies an admissible matrix solution of the Hamiltonian system. The $M$-matrix is therefore given by

$$
M(\lambda)=-U(0) V(0)^{-1}=\left(\begin{array}{cc}
-y_{1}(0) / y_{1}^{\prime}(0) & 0 \\
0 & -y_{2}(0) / y_{2}^{\prime}(0)
\end{array}\right) .
$$


We now recognise that

$$
M(\lambda)=\operatorname{diag}\left(m_{1}(\lambda), m_{2}(\lambda)\right)
$$

where $m_{1}$ and $m_{2}$ are, respectively, the scalar $m$-functions associated with the Sturm-Liouville equations

$$
-y^{\prime \prime}+x y=\lambda y, \quad-y^{\prime \prime}-x y=\lambda y, \quad x \in(0, \infty) .
$$

The conditions for a HELP inequality reduce to the corresponding conditions for each of these two scalar equations.

For the first equation there is only point spectrum. The first few eigenvalues, subject to the Neumann boundary condition $y^{\prime}(0)=0$, are given approximately by

$$
\lambda_{0} \approx 1.01879297, \quad \lambda_{1} \approx 10.5507875, \quad \lambda_{2} \approx 23.2333564 .
$$

(These were obtained using the code SL02F [14].) The equation $-y^{\prime \prime}+x y=\lambda y$ has an associated 'shifted' HELP inequality of the form (2) for $\tau=\lambda_{k}, k=0,1,2, \ldots$ For the second equation, the whole real line consists of continuous spectrum, and there is a HELP inequality [6, Example 5] of the form (2) for any value of $\tau$. Thus for the equation (62) there is no HELP inequality, but there are HELP inequalities associated with each of the 'shifted' equations

$$
-Y^{\prime \prime}+\left\{\left(\begin{array}{cc}
x & 0 \\
0 & -x
\end{array}\right)+\lambda_{k} I\right\} Y=\lambda Y, \quad x \in(0, \infty), \quad k=0,1,2, \ldots
$$

As an aside we observe that for (62) there is continuous spectrum on the whole real line with discontinuities of the spectral function at the eigenvalues of the equation $-y^{\prime \prime}+x y=\lambda y$ with boundary condition $y^{\prime}(0)=0$.

\subsection{Example 3}

We consider a fourth-order matrix-vector Sturm-Liouville problem

$$
Y^{(\mathrm{iv})}+\left(\begin{array}{cc}
q_{1}(x) & 0 \\
0 & q_{2}(x)
\end{array}\right) Y=\lambda Y, \quad x \in(0, \infty) .
$$


We convert this into a Hamiltonian system by defining

$$
U=\left(\begin{array}{c}
Y \\
Y^{\prime}
\end{array}\right), \quad V=\left(\begin{array}{c}
-Y^{\prime \prime \prime} \\
Y^{\prime \prime}
\end{array}\right)
$$

so that

$$
\begin{aligned}
-V^{\prime} & =\left(\begin{array}{cccc}
\lambda-q_{1}(x) & 0 & 0 & 0 \\
0 & \lambda-q_{2}(x) & 0 & 0 \\
0 & 0 & 0 & 0 \\
0 & 0 & 0 & 0
\end{array}\right) U+\left(\begin{array}{llll}
0 & 0 & 0 & 0 \\
0 & 0 & 0 & 0 \\
1 & 0 & 0 & 0 \\
0 & 1 & 0 & 0
\end{array}\right) V, \\
U^{\prime} & =\left(\begin{array}{llll}
0 & 0 & 1 & 0 \\
0 & 0 & 0 & 1 \\
0 & 0 & 0 & 0 \\
0 & 0 & 0 & 0
\end{array}\right) U+\left(\begin{array}{llll}
0 & 0 & 0 & 0 \\
0 & 0 & 0 & 0 \\
0 & 0 & 1 & 0 \\
0 & 0 & 0 & 1
\end{array}\right) V .
\end{aligned}
$$

Once again it is easy to check Assumptions 1-4 and it is also easy to see that $D(f, f)$ is equivalent to the usual Dirichlet form associated with (63).

Clearly the original fourth order equation (63) can be decoupled into two scalar fourth order equations. Under suitable limit-point hypotheses, let $y_{1}$ and $z_{1}$ denote linearly independent $L^{2}$ solutions of

$$
y^{(\mathrm{iv})}+q_{1}(x) y=\lambda y,
$$

and let $y_{2}$ and $z_{2}$ denote linearly independent $L^{2}$ solutions of

$$
y^{(\mathrm{iv})}+q_{2}(x) y=\lambda y .
$$

We can then form four admissible solutions for our Hamiltonian system:

$$
\begin{aligned}
& f_{1}(x)^{\mathrm{T}}=\left(y_{1}(x), 0, y_{1}^{\prime}(x), 0,-y_{1}^{\prime \prime \prime}(x), 0, y_{1}^{\prime \prime}(x), 0\right), \\
& f_{2}(x)^{\mathrm{T}}=\left(z_{1}(x), 0, z_{1}^{\prime}(x), 0,-z_{1}^{\prime \prime \prime}(x), 0, z_{1}^{\prime \prime}(x), 0\right), \\
& f_{3}(x)^{\mathrm{T}}=\left(0, y_{2}(x), 0, y_{2}^{\prime}(x), 0,-y_{2}^{\prime \prime \prime}(x), 0, y_{2}^{\prime \prime}(x)\right), \\
& f_{4}(x)^{\mathrm{T}}=\left(0, z_{2}(x), 0, z_{2}^{\prime}(x), 0,-z_{2}^{\prime \prime \prime}(x), 0, z_{2}^{\prime \prime}(x)\right) .
\end{aligned}
$$


The $4 \times 4 M$-matrix is thus given by

$$
\begin{aligned}
M(\lambda)= & -\left(\begin{array}{cccc}
y_{1}(0) & z_{1}(0) & 0 & 0 \\
0 & 0 & y_{2}(0) & z_{2}(0) \\
y_{1}^{\prime}(0) & z_{1}^{\prime}(0) & 0 & 0 \\
0 & 0 & y_{2}^{\prime}(0) & z_{2}^{\prime}(0)
\end{array}\right) \\
& \times\left(\begin{array}{cccc}
-y_{1}^{\prime \prime \prime}(0) & -z_{1}^{\prime \prime \prime}(0) & 0 & 0 \\
0 & 0 & -y_{2}^{\prime \prime \prime}(0) & -z_{2}^{\prime \prime \prime}(0) \\
y_{1}^{\prime \prime}(0) & z_{1}^{\prime \prime}(0) & 0 & 0 \\
0 & 0 & y_{2}^{\prime \prime}(0) & z_{2}^{\prime \prime}(0)
\end{array}\right)^{-1} .
\end{aligned}
$$

This gives

$$
M(\lambda)=P\left(\begin{array}{cc}
M_{1}(\lambda) & \mathbf{0} \\
\mathbf{0} & M_{2}(\lambda)
\end{array}\right) P^{-1}
$$

where 0 denotes the $2 \times 2$ zero matrix, $M_{j}(\lambda)(j=1,2)$ denote the $2 \times 2$ Titchmarsh-Weyl matrices

$$
M_{j}(\lambda)=-\left(\begin{array}{ll}
y_{j}(0) & z_{j}(0) \\
y_{j}^{\prime}(0) & z_{j}^{\prime}(0)
\end{array}\right)\left(\begin{array}{cc}
-y_{j}^{\prime \prime \prime}(0) & z_{j}^{\prime \prime \prime}(0) \\
y_{j}^{\prime \prime}(0) & z_{j}^{\prime \prime}(0)
\end{array}\right)^{-1}
$$

and $P$ is the $4 \times 4$ permutation matrix

$$
P=\left(\begin{array}{llll}
1 & 0 & 0 & 0 \\
0 & 0 & 1 & 0 \\
0 & 1 & 0 & 0 \\
0 & 0 & 0 & 1
\end{array}\right)
$$

The matrices $M_{1}$ and $M_{2}$ are Titchmarsh-Weyl matrices for the fourth order problems

$$
y^{(\mathrm{iv})}+q_{1}(x) y=\lambda y, \quad y^{(\mathrm{iv})}+q_{2}(x) y=\lambda y .
$$

Thus, as in the case of Example 2, the conditions for a HELP inequality in Theorem 4.5 become equivalent to the conditions for both of the scalar equations in (64) to have associated HELP inequalities. 
The coefficient matrix

$$
Q(x)=\left(\begin{array}{cc}
q_{1}(x) & 0 \\
0 & q_{2}(x)
\end{array}\right)
$$

in (63) can be replaced by any matrix of the form $R Q(x) R^{\mathrm{T}}$ where $R$ is orthogonal and constant, and the same result will hold: the conditions for a HELP inequality for the matrix-vector system reduce to the conditions for HELP inequalities for the associated scalar problems.

\section{CONCLUDING REMARKS}

In this paper we have generalised the work of Dias to Hamiltonian systems. In so doing, we have been forced to abandon the natural Hilbert-space setting which Dias uses for the $2 n$th order scalar selfadjoint case; in particular, therefore, our formula for the so-called Von Neumann decomposition has had to be proved in a very direct way.

\section{References}

[1] F.V. Atkinson. Discrete and Continuous Boundary Value Problems. Academic Press, New York and London, 1964.

[2] B.M. Brown and N.G.J. Dias. The HELP type integral inequalities for $2 n$th order differential operators. In General Inequalities, 7 (Oberwolfach, 1995), volume 123 of Internat. Ser. Numer. Math., pp. 179-192. Birkhäuser, Basel, 1997.

[3] B.M. Brown, M.S.P. Eastham and D.K.R. McCormack. A new algorithm for computing the spectral matrix for higher-order differential equations and the location of discrete eigenvalues. In Proceedings of the AMS/Siam meeting on the foundations of numerical analysis, Park City USA July 1995, 1996.

[4] B.M. Brown, W.D. Evans and V.G. Kirby. A numerical investigation of HELP inequalities. Results in Mathematics, 25: 20-39, 1994.

[5] N.G.J. Dias. On an integral inequality associated with a $2 n$th order quasi-differential expression. Ph.D. thesis, Cardiff: University of Wales, 1994.

[6] W.D. Evans and W.N. Everitt. A return to the Hardy-Littlewood inequality. Proc. Roy. Soc. Lond, A. 380: 447-486, 1982.

[7] W.D. Evans and W.N. Everitt. HELP inequalities for limit-circle and regular problems. Proc. Roy. Soc. Lond., A 432: 367-390, 1991.

[8] W.D. Evans and A. Zettl. Norm inequalities involving derivatives. Proc. Roy. Soc. Lond., A 82: 51-70, 1978.

[9] W.N. Everitt. On an extension to an integro-differential inequality of Hardy, Littlewood and Polya. Proc. Roy. Soc. Edin., A: 295-333. 1971/72.

[10] G.H. Hardy and J.E. Littlewood. Some inequalities connected with the calculus of variations. Quart. J. Math, 2(3): 241-252, 1932.

[11] G.H. Hardy, J.E. Littlewood and G. Polya. Inequalities. Cambridge University Press., 1934. 
[12] D.B. Hinton and J.K. Shaw. On Titchmarsh-Weyl $m(\lambda)$-functions for Hamiltonian systems. J. Diff. Equations, 40: 316-342, 1981.

[13] V. Hutson and J.S. Pym. Applications of functional analysis and operator theory, volume 146 of Mathematics in Science and Engineering. Academic Press Inc. (Harcourt Brace Jovanovich Publishers), New York, 1980.

[14] M. Marletta and J.D. Pryce. Automatic solution of Strum-Liouville problems using Pruess method. J. Comp. Appl. Math., 39: 57-58, 1992.

[15] William T. Reid. Sturmian Theory for Ordinary Differential Equations, volume 31 of Applied Mathematical Sciences. Springer-Verlag, New York, 1980. With a preface by John Burns.

[16] P.W. Walker. A vector-matrix formulation for formally symmetric ordinary differential equations with applications to solutions of integrable square. J. Lon. Math. Soc., 9: 151-159, 1974. 